\title{
Extrauterine growth restriction in extremely preterm infants based on the Intergrowth-21st Project Preterm Postnatal Follow-up Study growth charts and the Fenton growth charts
}

\author{
Yoo-jin Kim ${ }^{1,2} \cdot$ Seung Han Shin ${ }^{1,3}$ (D) Hannah Cho ${ }^{1,4} \cdot$ Seung Hyun Shin ${ }^{1} \cdot$ Seh Hyun Kim ${ }^{1} \cdot$ In Gyu Song ${ }^{1,5}$. \\ Ee-Kyung Kim ${ }^{1} \cdot$ Han-Suk Kim ${ }^{1}$
}

Received: 22 May 2020 / Revised: 6 August 2020 / Accepted: 26 August 2020 / Published online: 9 September 2020

(C) Springer-Verlag GmbH Germany, part of Springer Nature 2020

\begin{abstract}
Growth charts are essential for monitoring the postnatal growth of preterm infants. The preterm postnatal follow-up study (PPFS) of the Intergrowth-21st Project provides new growth standards based on a longitudinal study. This study was conducted to investigate the prevalence of extrautrine growth restriction (EUGR) and the associated factors of EUGR in preterm infants, using the PPFS charts and the Fenton charts. Data of 1,356 infants with gestational age (GA) less than 28 weeks from the Korean Neonatal Network were analysed. The prevalence of small for gestational age (SGA) of weight and length was higher with the Intergrowth charts than with the Fenton charts. EUGR in weight and length was more prevalent when using the Fenton charts. Multivariate analysis showed that low GA, high birthweight $z$ score, male, treated patent ductus arteriosus (PDA), necrotizing enterocolitis, intraventricular haemorrhage and duration of parenteral nutrition (PN) were associated with EUGR in weight by the Intergrowth charts. High birthweight $z$ score, treated PDA and PN duration were associated with EUGR defined by the Fenton charts.

Conclusion: Compared to the Fenton charts, SGA was more defined and EUGR was less prevalent in extremely low gestational infants, while EUGR defined by the Intergrowth charts categorized infants with adverse clinical courses more elaborately.

What is Known:

- Preterm infants are at risk of extrauterine growth restriction (EUGR), although optimal postnatal growth is important for the long-term outcomes.

- Growth charts are essential tools to monitor the postnatal growth of preterm infants.

What is New:

- EUGR of weight and length were less defined with the Intergrowth charts than the Fenton charts.

- EUGR defined by the Intergrowth preterm postnatal follow-up study (PPFS) chart categorized preterm infants with morbidities more elaborately than the Fenton charts.
\end{abstract}

Keywords Extrauterine growth restriction · Preterm · Very low birth weight infant $\cdot$ Intergrowth-21st Project

Communicated by Daniele De Luca

Electronic supplementary material The online version of this article (https://doi.org/10.1007/s00431-020-03796-0) contains supplementary material, which is available to authorized users.

Seung Han Shin

revival421@snu.ac.kr

Extended author information available on the last page of the article

\section{Introduction}

Preterm infants are at risk of extrauterine growth restriction (EUGR) [1], and optimal postnatal growth is important not only for survival but also for the long-term outcomes of preterm infants $[2,3]$. Despite recent improvements in the neonatal care of preterm infants, EUGR is still prevalent among premature babies during and after the neonatal intensive care unit (NICU) stay [4].

Growth charts are essential tools to monitor postnatal growth of preterm infants. The American Academy of 
Pediatrics (AAP) recommended that the growth rate of normal foetuses should be used as a growth standard for preterm infants. Estimated foetal weight charts based on intrauterine growth had been used as references for monitoring postnatal growth [5]. Another type of neonatal chart is based on birthweight for gestational age (GA) from various sample sizes ranging from a single hospital sample to a national population. The Fenton growth chart is one of the commonly used reference charts based on size at birth, developed from the Babson and Benda graph and several population studies [6-8]. As it is not based on the longitudinal study, the change in weight after birth in preterm population was not included in the data of the Fenton charts.

The Intergrowth-21st Project is a multicentre, multi-ethnic, population-based project. Of the five subjects included in the project, the Preterm Postnatal Follow-up Study (PPFS), was conducted to produce standards for postnatal growth in preterm infants as a more appropriate alternative to existing references based on size-at-birth measurements. Growth charts were generated based on rigorous and standardized anthropometric measurements of selected preterm infants from the original Intergrowth-21st Project [9]. However, the GA of preterm infants included in the PPFS was moderately preterm, and data for very preterm infants were obtained from the small sample size.

Based on the inherent conceptual and methodological limitations of these charts, there is currently no ideal growth standard for monitoring postnatal growth in preterm infants [10-12]. Significant deviations were found in the calculation of postnatal growth depending on the reference dataset that was used [13].

The objective of this study was to compare the prevalence of EUGR in extremely low GA infants who were registered in the Korean Neonatal Network (KNN) using the Fenton and PPFS charts and to investigate factors associated with EUGR by both charts.

\section{Methods}

This is a population-based study of preterm infants who were registered in the KNN between January 2013 and December 2015. The KNN is a nationwide neonatal network based on a prospective web-based registry for very low birth weight (VLBW) infants, which covers more than $70 \%$ of the total number of VLBW infants in Korea [14]. The KNN registry was approved by the institutional review board, and written informed consent was obtained from the parents upon enrolment at each participating hospital. The data in the KNN database comprises antenatal and perinatal histories, postnatal morbidities and clinical outcomes that were evaluated during and after the hospital stay using a standardised electronic casereport form. Among the registered population, preterm infants who were born at $<28$ weeks of gestation were included. Infants who were born at $<24$ weeks of gestation, had congenital anomalies and died or were transferred to other hospitals were excluded. For the Fenton growth charts available under 50 weeks of postmenstrual age (PMA), those who were not discharged from the hospital at 50 weeks of PMA were also excluded from the study.

Gestational age was calculated according to the last menstrual period. Respiratory distress syndrome (RDS) was defined by radiologic and clinical diagnosis with treatment with surfactant replacement. Bronchopulmonary dysplasia (BPD) was defined as the need for supplemental oxygen or ventilator support at 28 days after birth [15]. Necrotizing enterocolitis (NEC) was defined according to Bell's criteria (stage 2 or higher) [16]. Intraventricular haemorrhage (IVH) was defined by the Papile criteria, using cranial ultrasonography [17]. Retinopathy of prematurity (ROP) was defined according to the international classification of ROP [18].

Percentile and $z$ scores of weight, length and head circumference $(\mathrm{HC})$ at birth were calculated using both the Fenton growth charts and the Intergrowth-21st very preterm size charts (https://intergrowth21.tghn.org/standards-tools/) [7, $19,20]$. Size at discharge was also calculated using the Fenton and Intergrowth PPFS charts. Small for gestational age (SGA) was defined when weight, length and $\mathrm{HC}$ were less than the 10th percentile for GA [21]. The first definition of EUGR was decreased $z$ scores of weight, length and HC > 1 at discharge compared with $z$ scores at birth [4]. The second definition of EUGR was weight, length and $\mathrm{HC}$ at discharge below the 10th percentile. The need for approval was waived by the Institutional Review Board of Seoul National University Hospital.

Statistical analysis was performed with STATA 12.1 (StataCorp. 2011, College Station, TX, USA). The paired $t$ test was used to compare $z$ scores according to the different growth charts at birth and discharge. Cochran's $Q$ test was used to compare the prevalence of EUGR in the study population according to the two growth charts. Student's $t$ test and Fisher's exact test were conducted to compare factors between the EUGR and non-EUGR groups defined by decreased $z$ score $>1$ at discharge according to the two growth charts. Multivariate logistic regression analysis for EUGR in weight defined by decrease $z$ score $>1$ was conducted in each growth chart to determine factors associated with EUGR. All possible regressions were conducted with covariates based on the $p$ value $(p<0.10)$ in the univariate analysis. The variance inflation factor (VIF) was calculated to treat multicollinearity and covariates with VIF $>10$ were not included in the multiple logistic regression. Goodness of fit was evaluated by Hosmer-Lemeshow test. Sensitivity analysis of multivariate linear regressions was performed for changes in $z$ score of weight as continuous variables and provided in the supplementary materials. 


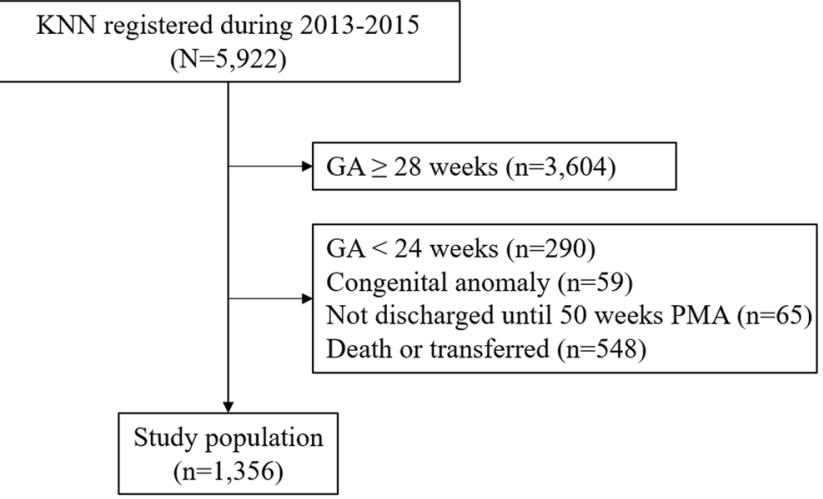

Fig. 1 Selection of study population. GA, gestational age; PMA, postmenstrual age

\section{Results}

Among the 5,922 preterm infants registered in the KNN database during the study period, 2,318 infants with GA $<28$ weeks were selected (Fig. 1). Among them, 290 infants who were born at $<24$ weeks of gestation, 59 infants with a congenital anomaly and 65 infants who were not discharged until 50 weeks of PMA were excluded. After additional exclusion of 548 infants who died or were transferred to other hospitals, 1,356 infants remained in the study population.

The GA at birth and PMA at discharge were $26.4 \pm 1.1$ and $39.9 \pm 3.2$ weeks (Table 1). Weights at birth and discharge were $899.6 \pm 189.7$ and $2824.2 \pm 637.3$ grams, respectively. The neonatal morbidities of the study population are provided in the supplementary materials (Table S2).

SGA of weight, length and $\mathrm{HC}$ at birth were more prevalent with the Intergrowth chart (Table 2). z-scores of weight,

Table 1 Perinatal characteristics and body size of study population

\begin{tabular}{ll}
\hline & $\begin{array}{l}\text { Study population } \\
(n=1,356)\end{array}$ \\
\hline Gestational age (weeks) & $26.4 \pm 1.1$ \\
Birthweight (grams) & $899.6 \pm 189.7$ \\
Birth length (cm) & $34.3 \pm 2.6$ \\
Birth head circumference (cm) & $24.1 \pm 1.7$ \\
Male sex & $701(51.7)$ \\
Maternal diabetes mellitus & $97(7.2)$ \\
Oligohydramnios & $151(12.3)$ \\
Preterm premature rupture of membrane & $584(43.4)$ \\
Multiple birth & $404(29.8)$ \\
Maternal hypertension & $143(10.6)$ \\
Postmenstrual age at discharge (weeks) & $39.9 \pm 3.2$ \\
Weight at discharge (grams) & $2824.2 \pm 637.3$ \\
Length at discharge (cm) & $46.1 \pm 3.5$ \\
Head circumference at discharge (cm) & $32.9 \pm 2.1$ \\
\hline
\end{tabular}

Values are expressed as number $(\%)$ or mean \pm standard deviation
Table 2 Size at birth and at discharge by the Fenton and Intergrowth charts

\begin{tabular}{llll}
\hline & Fenton & Intergrowth & $p$ value \\
\hline SGA weight, $n(\%)$ & $88(6.4)$ & $109(8)$ & $<0.001$ \\
SGA length, $n(\%)$ & $92(7.1)$ & $137(10.5)$ & $<0.001$ \\
SGA HC, $n(\%)$ & $94(7.3)$ & $119(9.3)$ & $<0.001$ \\
Birth weight $z$ score & $0.22 \pm 0.85$ & $0.18 \pm 0.93$ & $<0.001$ \\
Birth length $z$ score & $0.18 \pm 0.97$ & $-0.3 \pm 0.84$ & $<0.001$ \\
Birth HC $z$ score & $0.17 \pm 1.05$ & $-0.18 \pm 0.9$ & $<0.001$ \\
Discharge weight $z$ score & $-1.44 \pm 1.21$ & $-1.03 \pm 1.33$ & $<0.001$ \\
Discharge length $z$ score & $-1.94 \pm 1.46$ & $-2.1 \pm 1.92$ & $<0.001$ \\
Discharge HC $z$ score & $-1.25 \pm 1.28$ & $-1.53 \pm 1.76$ & $<0.001$ \\
${ }^{\S} z$ score weight & $-1.67 \pm 1.05$ & $-1.21 \pm 1.15$ & $<0.001$ \\
${ }^{\S} z$ score length & $-2.08 \pm 1.26$ & $-1.76 \pm 1.64$ & $<0.001$ \\
${ }^{\S} z$ score HC & $-1.38 \pm 1.33$ & $-1.31 \pm 1.66$ & $<0.001$ \\
\hline
\end{tabular}

Values are expressed as number $(\%)$ or mean \pm standard deviation. $S G A$, small for gestational age; $H C$, head circumference. ${ }^{\S}$ Differences between values at discharge and at birth

length and $\mathrm{HC}$ at birth, as well as at discharge, were lower with Intergrowth charts, except for the weight $z$ score at discharge (Fenton $-1.44 \pm 1.21$ vs. Intergrowth $-1.03 \pm 1.33$ ).

a

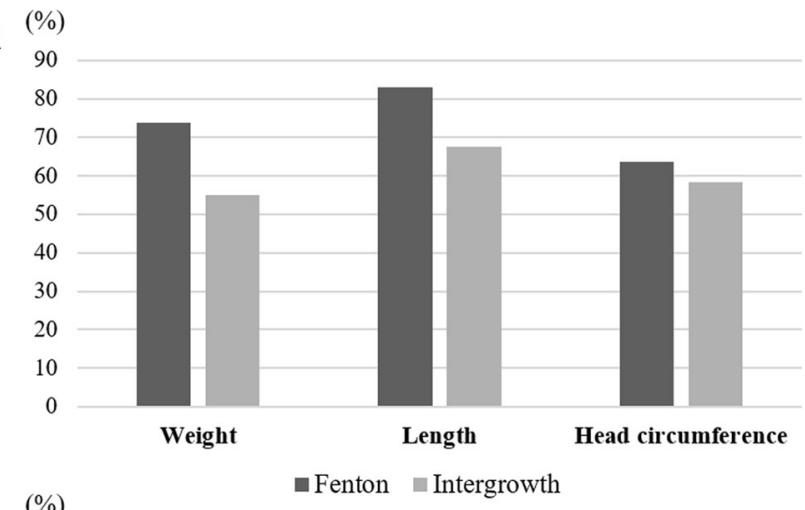

b $(\%)$

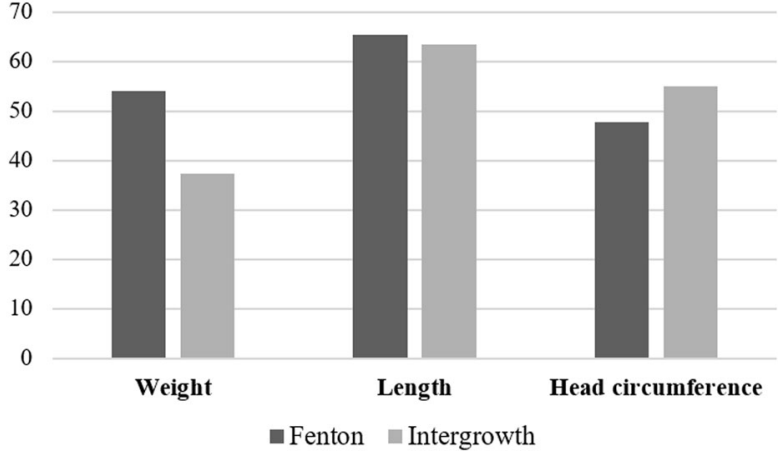

Fig. 2 Prevalence of EUGR according to the growth charts. EUGR of weight, length and $\mathrm{HC}$ by the Fenton and Intergrowth charts when EUGR was defined as a decrease in $z$ score $>1$ at discharge (a) and when EUGR was defined as body size $<10$ th percentile at discharge (b). Cochran's $Q$ test showed that the $p$ value of all comparison of EUGR between the Fenton and Intergrowth chart were $<0.001$. EUGR, extrauterine growth restriction 
Table 3 Clinical characteristics of study population by EUGR in weight based on the Intergrowth charts

\begin{tabular}{|c|c|c|c|}
\hline & Non-EUGR $(n=612)$ & $\begin{array}{l}\text { EUGR } \\
(n=755)\end{array}$ & $p$ value \\
\hline Gestational age (weeks) & $26.6 \pm 1.0$ & $26.2 \pm 1.0$ & $<0.001$ \\
\hline Birthweight (grams) & $891 \pm 201.1$ & $908.7 \pm 178.9$ & 0.087 \\
\hline Birthweight $z$ score & $0.0 \pm 1.1$ & $0.3 \pm 0.8$ & $<0.001$ \\
\hline Male sex & $275(45.2)$ & $426(57.0)$ & $<0.001$ \\
\hline Caesarean section & $428(70.3)$ & $532(71.2)$ & 0.719 \\
\hline Maternal diabetes mellitus & $49(8.1)$ & $48(6.4)$ & 0.289 \\
\hline Chorioamnionitis & $240(44.9)$ & $285(46.6)$ & 0.593 \\
\hline PPROM & $246(40.8)$ & $338(45.6)$ & 0.086 \\
\hline Oligohydramnios & $71(13.0)$ & $80(11.7)$ & 0.541 \\
\hline Prenatal steroid & $488(81.1)$ & $600(82.1)$ & 0.541 \\
\hline Multiple birth & $184(30.2)$ & $220(29.5)$ & 0.766 \\
\hline Hypertension & $86(14.1)$ & $57(7.6)$ & $<0.001$ \\
\hline Apgar score $1 \mathrm{~min}$ & $4 \pm 1.9$ & $3.8 \pm 1.7$ & 0.102 \\
\hline Apgar score $5 \mathrm{~min}$ & $6.3 \pm 1.8$ & $6.3 \pm 1.7$ & 0.714 \\
\hline RDS & $587(96.4)$ & $736(98.5)$ & 0.013 \\
\hline Treated PDA & $319(52.4)$ & $461(61.7)$ & 0.001 \\
\hline Moderate to severe BPD & $267(43.8)$ & $367(49.1)$ & 0.056 \\
\hline $\mathrm{IVH} \geq$ grade 3 & $36(5.9)$ & $105(14.1)$ & $<0.001$ \\
\hline NEC & $20(3.3)$ & $74(9.9)$ & $<0.001$ \\
\hline ROP $\geq$ stage 2 & $126(20.7)$ & $207(27.8)$ & 0.003 \\
\hline Sepsis & $163(26.8)$ & $249(33.3)$ & 0.009 \\
\hline Duration of PN (days) & $29.5 \pm 17.7$ & $41.1 \pm 23.0$ & $<0.001$ \\
\hline Length of stay (days) & $90.4 \pm 23.7$ & $98.7 \pm 23.8$ & $<0.001$ \\
\hline
\end{tabular}

Values are expressed as number $(\%)$ or mean \pm standard deviation. EUGR, extrauterine growth restriction; $P P R O M$, preterm premature rupture of membrane; $R D S$, respiratory distress syndrome; $P D A$, patent ductus arteriosus; $B P D$, bronchopulmonary dysplasia; $I V H$, intraventricular haemorrhage; $N E C$, necrotizing enterocolitis; $R O P$, retinopathy of prematurity; $P N$, parenteral nutrition
However, differences in $z$ scores in all body measurements at discharge from at birth were smaller when the Intergrowth charts were used.

When EUGR was defined as a decrease in $z$ score by more than one, the prevalence of EUGR of weight, length and HC by the Fenton charts were higher than those defined by the Intergrowth charts (Fig. 2). EUGR defined by less than the 10th percentile at discharge of weight and length by the Fenton charts also were higher than those defined by the Intergrowth charts. However, EUGR of $\mathrm{HC}$ by the Fenton charts was less prevalent than that of the Intergrowth charts.

Infants with EUGR defined as decreased $z$ score $>1$ according to the Intergrowth charts had lower GA $(26.6 \pm 1.0 \mathrm{vs}$. $26.2 \pm 1.0$ weeks, $p<0.001)$ and higher birthweight $z$ score $(0.0 \pm 1.1$ vs. $0.3 \pm 0.8, p<0.001)$ than with infants with nonEUGR (Table 3). Male sex, RDS, treated PDA, IVH grade $\geq$ 3 , NEC, ROP stage $\geq 2$ and sepsis were more prevalent in the EUGR group.
When EUGR was defined as decreased $z$ core $>1$ by the Fenton charts, GA was lower $(26.6 \pm 1.1$ vs. $26.3 \pm$ 1.0 weeks, $p<0.001)$ and birthweight $z$ score was higher in the EUGR group $(-0.1 \pm 1.0$ vs. $0.3 \pm 0.9$, $p<0.001$ ) (Table 4). There were more cases of maternal hypertension in the non-EUGR group. RDS, treated PDA, IVH, NEC and ROP were more prevalent in the EUGR group.

Multivariate logistic regression was conducted to find associated factors with EUGR. When EUGR was defined by decreased $z$ score $>1$ based on the Intergrowth charts, lower GA, higher birthweight z-score, male sex, high grade IVH, NEC and longer duration of parenteral nutrition (PN) were associated with EUGR (goodness of fit, $p=0.426$ ) (Table 5). When the same definition of EUGR was made by the Fenton charts, higher birthweight $z$ score, treated PDA, and longer duration of PN were associated with EUGR (goodness of fit, $p=0.286$ ). 
Table 4 Clinical characteristics of the study population by EUGR in weight based on the Fenton charts

\begin{tabular}{llll}
\hline & $\begin{array}{l}\text { Non-EUGR } \\
(n=355)\end{array}$ & $\begin{array}{l}\text { EUGR } \\
(n=1,001)\end{array}$ & $p$ value \\
\hline Gestational age (weeks) & $26.6 \pm 1.1$ & $26.3 \pm 1.0$ & $<0.001$ \\
Birthweight (grams) & $880.2 \pm 202.4$ & $908 \pm 184$ & 0.017 \\
Birthweight $z$ score & $-0.1 \pm 1.0$ & $0.3 \pm 0.9$ & $<0.001$ \\
Male sex & $175(49.3)$ & $526(52.6)$ & 0.294 \\
Caesarean section & $251(70.7)$ & $709(70.8)$ & 0.720 \\
Maternal diabetes mellitus & $30(8.5)$ & $67(6.7)$ & 0.281 \\
Chorioamnionitis & $133(42.9)$ & $392(46.9)$ & 0.231 \\
PPROM & $157(44.7)$ & $427(43.0)$ & 0.573 \\
Oligohydramnios & $44(13.8)$ & $107(11.8)$ & 0.372 \\
Prenatal steroid & $286(81.7)$ & $802(81.6)$ & 1.000 \\
Multiple birth & $112(31.6)$ & $292(29.2)$ & 0.418 \\
Hypertension & $56(15.8)$ & $87(8.7)$ & $<0.001$ \\
Apgar score 1 min & $4.0 \pm 1.9$ & $3.9 \pm 1.7$ & 0.264 \\
Apgar score 5 min & $6.3 \pm 1.8$ & $6.3 \pm 1.7$ & 0.463 \\
RDS & $340(95.8)$ & $983(98.2)$ & 0.015 \\
Treated PDA & $170(47.9)$ & $610(60.9)$ & $<0.001$ \\
Moderate to severe BPD & $170(47.9)$ & $464(46.4)$ & 0.621 \\
IVH $\geq$ grade 3 & $19(5.4)$ & $122(12.2)$ & $<0.001$ \\
NEC & $14(3.9)$ & $80(8.0)$ & 0.010 \\
ROP $\geq$ stage 2 & $73(20.6)$ & $260(26.0)$ & 0.045 \\
Sepsis & $93(26.2)$ & $319(31.9)$ & 0.051 \\
Duration of PN (days) & $28.1 \pm 16.9$ & $38.6 \pm 22.4$ & $<0.001$ \\
Length of stay (days) & $91.4 \pm 23.9$ & $96.2 \pm 24.0$ & 0.001 \\
\hline & & & \\
\hline
\end{tabular}

Values are expressed as number (\%) or mean \pm standard deviation. $E U G R$, extrauterine growth restriction; $P P R O M$, preterm premature rupture of membrane; $R D S$, respiratory distress syndrome; $P D A$, patent ductus arteriosus; $B P D$, bronchopulmonary dysplasia; $I V H$, intraventricular haemorrhage; $N E C$, necrotizing enterocolitis; $R O P$, retinopathy of prematurity; $P N$, parenteral nutrition

\section{Discussion}

The present study explored the prevalence and associated factors of EUGR in extremely low GA infants. EUGR defined as a $>1$ decrease in $z$ score in weight, length and $\mathrm{HC}$ as well as EUGR defined as $<10$ th percentile at discharge in weight and length were more prevalent based on the Fenton charts. When EUGR was defined as a $>1$ decrease in $z$ score in weight, the multivariate analysis showed that lower GA, higher birthweight $z$ score, male sex, IVH, NEC, and longer duration of PN were associated with EUGR by the Intergrowth charts, whereas EUGR by the Fenton charts showed association with higher birthweight $z$ score, treated PDA and longer duration of PN.
EUGR is a prevalent phenomenon during NICU stay in preterm infants. Clark et al. defined EUGR as below the 10th percentile at the time of discharge according to an intrauterine growth chart and reported the incidence of EUGR in weight and length at discharge to be $28 \%$ and $34 \%$ among preterm infants born at 23-34 weeks GA [21]. Vermont Oxford Network study reported that EUGR below the 10th percentile of weight by the Fenton growth chart occurred in half of VLBW infants [22]. The definition of EUGR as the change in $z$ scores also has been used, and a study using the Fenton chart reported the prevalence of EUGR during NICU admission in preterm infants born at GA of 22-32 weeks as $47 \%$ in 2005 and 38\% in 2012 [4, 23]. EUGR defined by the Fenton chart in the present study seemed higher than those reports, but the study population was less mature in our study compared with those in the aforementioned studies.

Studies comparing the Fenton and Intergrowth charts reported that EUGR for weight and length were less prevalent with the Intergrowth charts, as shown in the current study [24, 25]. To evaluate the appropriateness of the growth charts in defining EUGR, the comparison of long-term outcomes should be required, which was not investigated in the previous studies or in our study either. Rather, noted factors or morbidities associated with growth restriction in preterm infants such as low GA, IVH and NEC were associated with EUGR when defined according to the Intergrowth charts, but when defined by the Fenton charts, although treated PDA was associated with EUGR, low GA, IVH and NEC were not associated with EUGR [4, 21]. Linear regression analysis showed that NEC was associated with decreased weight $z$ score at discharge by Fenton charts, but more factors were associated with EUGR when it was defined by the Intergrowth charts (Table S3).

Growth charts based on foetal growth standards or crosssectional size-at-birth measurements by GA should be used prudently because growth in utero and ex utero is not comparable due to different biological processes as well as environmental and nutritional aspects. There have been concerns on the overestimation of EUGR in preterm infants because efforts to avoid EUGR such as aggressive nutritional support could lead to unfavourable outcomes later in life including insulin resistance [26, 27]. Intergrowth PPFS charts could be better standards for preterm infants because they were made during the postnatal period based on regular follow-up of preterm infants without adverse perinatal factors that could affect postnatal growth. As a result, the growth centiles in the PPFS charts were lower across the entire GA range than those in growth charts based on measurements at birth [9].

There are several limitations to our study. First, as only body sizes at birth and at discharge were registered in the database, information on growth patterns such as weight loss during first few days after birth and body sizes at specified timing (i.e. PMA 
Table 5 Factors associated with EUGR in weight based on the Intergrowth and Fenton charts

\begin{tabular}{|c|c|c|c|c|c|c|c|c|}
\hline & & \multicolumn{3}{|c|}{ Univariate analysis } & \multicolumn{4}{|c|}{ Multivariate analysis } \\
\hline & & OR & $95 \% \mathrm{CI}$ & $p$ value & Adjusted $\mathrm{OR}^{\S}$ & $95 \% \mathrm{CI}$ & $p$ value & VIF \\
\hline \multirow[t]{13}{*}{ Intergrowth } & Gestational age (weeks) & 0.73 & $(0.65-0.81)$ & $<0.001$ & 0.84 & $(0.74-0.95)$ & 0.006 & 7.440 \\
\hline & Birthweight $z$ score & 1.50 & $(1.33-1.70)$ & $<0.001$ & 1.65 & $(1.35-2.02)$ & $<0.001$ & 2.410 \\
\hline & SGA weight & 0.33 & $(0.22-0.50)$ & $<0.001$ & 0.81 & $(0.41-1.58)$ & 0.537 & 2.520 \\
\hline & Male sex & 1.61 & $(1.30-2.00)$ & $<0.001$ & 1.77 & $(1.39-2.24)$ & $<0.001$ & 2.110 \\
\hline & PPROM & 1.21 & $(0.98-1.51)$ & 0.080 & 1.04 & $(0.81-1.33)$ & 0.761 & 1.890 \\
\hline & Maternal hypertension & 0.50 & $(0.35-0.72)$ & $<0.001$ & 0.98 & $(0.62-1.53)$ & 0.923 & 1.510 \\
\hline & Moderate to severe BPD & 1.24 & $(1.00-1.53)$ & 0.052 & 0.80 & $(0.62-1.03)$ & 0.089 & 2.170 \\
\hline & Treated PDA & 1.47 & $(1.18-1.82)$ & 0.001 & 1.19 & $(0.93-1.52)$ & 0.176 & 2.540 \\
\hline & $\mathrm{IVH} \geq$ grade 3 & 2.60 & $(1.75-3.86)$ & $<0.001$ & 1.78 & $(1.16-2.74)$ & 0.008 & 1.170 \\
\hline & NEC & 3.24 & $(1.95-5.37)$ & $<0.001$ & 2.01 & $(1.13-3.56)$ & 0.017 & 1.170 \\
\hline & ROP $\geq$ stage 2 & 1.47 & $(1.14-1.89)$ & 0.003 & 1.15 & $(0.85-1.55)$ & 0.371 & 1.450 \\
\hline & Sepsis & 1.37 & $(1.08-1.73)$ & 0.009 & 0.89 & $(0.68-1.17)$ & 0.422 & 1.620 \\
\hline & Duration of PN (days) & 1.03 & $(1.02-1.04)$ & $<0.001$ & 1.03 & $(1.02-1.04)$ & $<0.001$ & 5.050 \\
\hline \multirow[t]{10}{*}{ Fenton } & Gestational age (weeks) & 0.80 & $(0.71-0.91)$ & $<0.001$ & 0.95 & $(0.83-1.09)$ & 0.454 & 5.180 \\
\hline & Birthweight $z$ score & 1.56 & $(1.35-1.80)$ & $<0.001$ & 1.86 & $(1.50-2.32)$ & $<0.001$ & 1.890 \\
\hline & SGA weight & 0.41 & $(0.26-0.63)$ & $<0.001$ & 1.10 & $(0.58-2.06)$ & 0.774 & 1.780 \\
\hline & Maternal hypertension & 0.51 & $(0.35-0.73)$ & $<0.001$ & 0.89 & $(0.57-1.37)$ & 0.587 & 1.390 \\
\hline & Treated PDA & 1.70 & $(1.33-2.17)$ & $<0.001$ & 1.35 & $(1.03-1.75)$ & 0.027 & 2.500 \\
\hline & $\mathrm{IVH} \geq$ grade 3 & 2.45 & $(1.49-4.04)$ & $<0.001$ & 1.66 & $(0.98-2.81)$ & 0.062 & 1.160 \\
\hline & NEC & 2.12 & $(1.18-3.78)$ & 0.012 & 1.24 & $(0.66-2.33)$ & 0.502 & 1.170 \\
\hline & ROP $\geq$ stage 2 & 1.35 & $(1.01-1.81)$ & 0.044 & 1.11 & $(0.80-1.56)$ & 0.527 & 1.420 \\
\hline & Sepsis & 1.32 & $(1.00-1.73)$ & 0.046 & 0.86 & $(0.64-1.17)$ & 0.341 & 1.610 \\
\hline & Duration of PN (days) & 1.03 & $(1.02-1.04)$ & $<0.001$ & 1.03 & $(1.02-1.04)$ & $<0.001$ & 4.740 \\
\hline
\end{tabular}

$\S$ Adjusted for factors associated with EUGR by each growth charts $(p<0.10)$ as shown in the table. EUGR, extrauterine growth restriction; SGA, small for gestational age; PPROM, preterm premature rupture of membrane; $B P D$, bronchopulmonary dysplasia; $P D A$, patent ductus arteriosus; $I V H$, intraventricular haemorrhage; $N E C$, necrotizing enterocolitis; $R O P$, retinopathy of prematurity; $P N$, parenteral nutrition; VIF, variance inflation factor

36 weeks) was not provided. Moreover, a quarter of eligible infants with gestational age $<28$ weeks were excluded because they died or were transferred to other hospitals [24, 25]. The other limitation was that long-term outcomes of growth and neurodevelopment were not investigated in the present study. Even though the prevalence of SGA and EUGR was changed by new growth charts, the more appropriate charts in extremely preterm infants could not be determined without long-term outcomes of the study population. However, this study analysed a large study population of nationwide registered data of preterm infants to evaluate the prevalence of EUGR and associated factors by the Fenton charts and the Intergrowth charts.

\section{Conclusions}

EUGR of weight and length were less prevalent with the Intergrowth charts than with the Fenton charts. More morbidities associated with growth restriction of weight were found when EUGR is defined by the Intergrowth charts. Despite the differences between two charts, we cannot conclude that one tool is better than the other, and further validation of Intergrowth charts with long-term outcome studies for extremely low GA infants might be required.

Authors' contributions Yoo-jin Kim and Seung Han Shin designed the study, performed data analysis, interpreted the data and drafted the manuscript. Hannah Cho, Seung Hyun Shin, In Gyu Song and Seh Hyun Kim performed data analysis and reviewed literatures. Ee-Kyung Kim and HanSuk Kim designed the study, monitored the study and revised the manuscript.

Funding This research was supported by funds from Research of Korea Centres for Disease Control and Prevention (2016-ER6307-02\#) and from the Basic Science Research Program through the National Research Foundation of Korea (NRF) funded by the Ministry of Education (2017R1D1A1B03036383).

\section{Compliance with ethical standards}

Conflict of interest The authors declare that they have no conflict of interest. 
Ethical approval This article does not contain any studies with human participants or animals performed by any of the authors.

Informed consent Informed consent was obtained from all individual participants included in the study.

Abbreviations EUGR, extrauterine growth restriction; NICU, Neonatal intensive care unit; $A A P$, American Academy of Pediatrics; PPFS, Preterm Postnatal Follow-up Study; $K N N$, Korean Neonatal Network; $V L B W$, very low birth weight; $P M A$, postmenstrual age; $R D S$, respiratory distress syndrome; $B P D$, bronchopulmonary dysplasia; $N E C$, necrotizing enterocolitis; $I V H$, intraventricular haemorrhage; $R O P$, retinopathy of prematurity; $H C$, Head circumference; $P N$, parenteral nutrition; $P D A$, patent ductus arteriosus; $G A$, gestational age; $S G A$, small for gestational age

\section{References}

1. Kavurt S, Celik K (2018) Incidence and risk factors of postnatal growth restriction in preterm infants. J Matern Fetal Neonatal Med 31:1105-1107

2. Lapillonne A, Griffin IJ (2013) Feeding preterm infants today for later metabolic and cardiovascular outcomes. J Pediatr 162:S7-S16

3. Embleton NE, Pang N, Cooke RJ (2001) Postnatal malnutrition and growth retardation: an inevitable consequence of current recommendations in preterm infants? Pediatrics 107:270-273

4. Griffin IJ, Tancredi DJ, Bertino E, Lee HC, Profit J (2016) Postnatal growth failure in very low birthweight infants born between 2005 and 2012. Arch Dis Child Fetal Neonatal Ed 101:F50-F55

5. American Academy of Pediatrics, Committee on Nutrition (1977) Nutritional needs of low-birth-weight infants. Pediatrics 60:519 530

6. Fenton TR (2003) A new growth chart for preterm babies: Babson and Benda's chart updated with recent data and a new format. BMC Pediatr 3:13

7. Fenton TR, Kim JH (2013) A systematic review and meta-analysis to revise the Fenton growth chart for preterm infants. BMC Pediatr 13:59

8. Babson SG, Benda GI (1976) Growth graphs for the clinical assessment of infants of varying gestational age. J Pediatr 89:814-820

9. Villar J, Giuliani F, Bhutta ZA, Bertino E, Ohuma EO, Ismail LC, Barros FC, Altman DG, Victora C, Noble JA, Gravett MG, Purwar M, Pang R, Lambert A, Papageorghiou AT, Ochieng R, Jaffer YA, Kennedy SH (2015) Postnatal growth standards for preterm infants: the Preterm Postnatal Follow-up Study of the INTERGROWTH-21 st Project. Lancet Glob Health 3:e681-e691

10. Villar J, Knight HE, de Onis M, Bertino E, Gilli G, Papageorghiou AT, Ismail LC, Barros FC, Bhutta ZA, International F, Newborn Growth C (2010) Conceptual issues related to the construction of prescriptive standards for the evaluation of postnatal growth of preterm infants. Arch Dis Child 95:1034-1038

11. Dudley NJ (2005) A systematic review of the ultrasound estimation of fetal weight. Ultrasound Obstet Gynecol 25:80-89

12. Giuliani F, Cheikh Ismail L, Bertino E, Bhutta ZA, Ohuma EO, Rovelli I, Conde-Agudelo A, Villar J, Kennedy SH (2016)
Monitoring postnatal growth of preterm infants: present and future. Am J Clin Nutr 103:635S-647S

13. Neubauer V, Fuchs T, Griesmaier E, Pupp-Peglow U, KiechlKohlendorfer U (2016) Comparing growth charts demonstrated significant deviations between the interpretation of postnatal growth patterns in very preterm infants. Acta Paediatr 105:268-273

14. Chang YS, Park HY, Park WS (2015) The Korean Neonatal Network: an overview. J Korean Med Sci 30(Suppl 1):S3-S11

15. Jobe AH, Bancalari E (2001) Bronchopulmonary dysplasia. Am J Respir Crit Care Med 163:1723-1729

16. Bell MJ (1978) Neonatal necrotizing enterocolitis. N Engl J Med 298:281-282

17. Papile LA, Munsick-Bruno G, Schaefer A (1983) Relationship of cerebral intraventricular hemorrhage and early childhood neurologic handicaps. J Pediatr 103:273-277

18. Garner A (1984) An international classification of retinopathy of prematurity. The Committee for the Classification of Retinopathy of Prematurity. Arch Ophthalmol 102:1130-1134

19. Villar J, Giuliani F, Fenton TR, Ohuma EO, Ismail LC, Kennedy SH, Consortium I-s (2016) INTERGROWTH-21st very preterm size at birth reference charts. Lancet 387:844-845

20. Chou JH, Roumiantsev S, Singh R (2020) PediTools electronic growth chart calculators: applications in clinical care research, and quality improvement. J Med Internet Res 22:e16204

21. Clark RH, Thomas P, Peabody J (2003) Extrauterine growth restriction remains a serious problem in prematurely born neonates. Pediatrics 111:986-990

22. Horbar JD, Ehrenkranz RA, Badger GJ, Edwards EM, Morrow KA, Soll RF, Buzas JS, Bertino E, Gagliardi L, Bellu R (2015) Weight Growth Velocity and Postnatal Growth Failure in Infants 501 to 1500 Grams: 2000-2013. Pediatrics 136:e84-e92

23. Zhang L, Li Y, Liang S, Liu XJ, Kang FL, Li GM (2019) Postnatal length and weight growth velocities according to Fenton reference and their associated perinatal factors in healthy late preterm infants during birth to term-corrected age: an observational study. Ital J Pediatr 45:1

24. Tuzun F, Yucesoy E, Baysal B, Kumral A, Duman N, Ozkan H (2018) Comparison of INTERGROWTH-21 and Fenton growth standards to assess size at birth and extrauterine growth in very preterm infants. J Matern Fetal Neonatal Med 31:2252-2257

25. Reddy KV, Sharma D, Vardhelli V, Bashir T, Deshbotla SK, Murki S (2019) Comparison of Fenton 2013 growth curves and Intergrowth-21 growth standards to assess the incidence of intrauterine growth restriction and extrauterine growth restriction in preterm neonates $</=32$ weeks. J Matern Fetal Neonatal Med 1-8

26. Singhal A, Fewtrell M, Cole TJ, Lucas A (2003) Low nutrient intake and early growth for later insulin resistance in adolescents born preterm. Lancet 361:1089-1097

27. Thureen PJ (2007) The neonatologist's dilemma: catch-up growth or beneficial undernutrition in very low birth weight infants-what are optimal growth rates? J Pediatr Gastroenterol Nutr 45(Suppl 3): S152-S154

Publisher's note Springer Nature remains neutral with regard to jurisdictional claims in published maps and institutional affiliations. 


\section{Affiliations}

\section{Yoo-jin Kim ${ }^{1,2} \cdot$ Seung Han Shin ${ }^{1,3}\left(\mathbb{D} \cdot\right.$ Hannah Cho ${ }^{1,4}$. Seung Hyun Shin ${ }^{1} \cdot$ Seh Hyun Kim ${ }^{1} \cdot$ In Gyu Song ${ }^{1,5}$. Ee-Kyung Kim ${ }^{1} \cdot$ Han-Suk Kim ${ }^{1}$}

Yoo-jin Kim

mercede@naver.com

Hannah Cho

lovevnhannah@naver.com

Seung Hyun Shin

siasince@hanmail.net

\section{Seh Hyun Kim}

drcorkim@gmail.com

1 Department of Paediatrics, Seoul National University College of Medicine, Seoul, Korea

2 Department of Pediatrics, Chungbuk National University Hospital, Cheongju-si, Republic of Korea

3 Department of Paediatrics, Seoul National University Children's Hospital, 101 Daehak-ro, Jongno-gu, Seoul 110-769, South Korea
In Gyu Song

pedigms@gmail.com

Ee-Kyung Kim

kimek@snu.ac.kr

Han-Suk Kim

kimhans@snu.ac.kr Department of Pediatrics, Korea University Anam Hospital, Seoul, Republic of Korea

5 Department of Pediatrics, Yonsei University College of Medicine, Seoul, Republic of Korea 